\title{
Procedure to count atoms with trustworthy single-atom sensitivity
}

\author{
S. Van Aert, ${ }^{*}$ A. De Backer, G. T. Martinez, B. Goris, S. Bals, and G. Van Tendeloo \\ EMAT, University of Antwerp, Groenenborgerlaan 171, B-2020 Antwerp, Belgium
}

\section{A. Rosenauer}

Institut für Festkörperphysik, Universität Bremen, Otto-Hahn-Allee 1, D-28359 Bremen, Germany

(Received 2 July 2012; revised manuscript received 6 February 2013; published 19 February 2013)

\begin{abstract}
We report a method to reliably count the number of atoms from high-angle annular dark field scanning transmission electron microscopy images. A model-based analysis of the experimental images is used to measure scattering cross sections at the atomic level. The high sensitivity of these measurements in combination with a thorough statistical analysis enables us to count atoms with single-atom sensitivity. The validity of the results is confirmed by means of detailed image simulations. We will show that the method can be applied to nanocrystals of arbitrary shape, size, and atom type without the need for a priori knowledge about the atomic structure.
\end{abstract}

Atomic resolution high-angle annular dark field (HAADF) scanning transmission electron microscopy (STEM) enables one to obtain projection images of the atomic columns of crystalline nanoparticles. These images are highly sensitive for the number of projected atoms in these columns. ${ }^{1,2}$ Therefore, this imaging technique is appealing to count the number of atoms in each column with single-atom sensitivity being of great importance to help determining the three-dimensional (3D) arrangement of all atoms. Previous quantification attempts often assume a linear dependence of the HAADF STEM intensities on the number of atoms using mass standards with known total number of atoms to calibrate this relation. ${ }^{3}$ More recently, LeBeau et al. ${ }^{4}$ suggested to quantify the number of atoms by matching normalized experimental intensities with image simulations. Although image simulations have been proven to match with experimental images within a 5\%-10\% error range, ${ }^{5,6}$ the validity of this approach is determined by the quality with which the detector and probe settings have been calibrated and the accuracy with which structure parameters including the Debye-Waller factor, surface strain relaxation, and static atomic displacements are taken into account. In general, the latter parameters are difficult to determine without knowing the structure a priori, hence limiting the practical use of image simulations to count the number of atoms. An alternative statistics-based method has been applied to quantify the number of atoms of a silver particle embedded in a stabilizing aluminum matrix. ${ }^{7}$ By combining atom counting results from two different viewing directions the 3D atomic structure has been reconstructed using so-called discrete tomography. In this article the method to count the number of atoms will be described in detail. The combination of a thorough statistical analysis with image simulations reveals trustworthy single-atom sensitivity. It will be shown that this approach can be applied to nanocrystals of arbitrary shape, size, and atom type without the need for a priori knowledge concerning the structure parameters.

The method we propose relies on an atomic column by atomic column quantification of the scattering cross sections. ${ }^{8}$ Therefore use is made of an empirical parametrized incoherent model describing the HAADF STEM image contrast. Based on this model, the total intensity $V_{i}$ can be quantified for each projected atomic column $i=1, \ldots, I$ using statistical parameter estimation theory. As an example, consider a HAADF STEM image of a $\mathrm{Pb}$ nanoparticle embedded in crystalline Si. This image, shown in Fig. 1(a), has been recorded using a double aberration-corrected FEI TITAN operated at $300 \mathrm{kV}$. The refined model, shown in Fig. 1(b), is in excellent agreement with the experimental data demonstrating the quality of the model used. The scattered intensities of all $\mathrm{Pb}$ columns can be used as a performance measure to count the number of $\mathrm{Pb}$ atoms within a column of $\mathrm{Si}$ atoms. Indeed, since the thickness of the sample can be assumed to be constant over the particle area, substitution of a $\mathrm{Si}$ atom by a $\mathrm{Pb}$ atom leads to an increase of the scattered intensity. Ideally, a histogram of scattered intensities would thus consist of isolated peaks, where each peak is generated by a set of columns having the same number of $\mathrm{Pb}$ atoms. In practice however, the peaks are smeared out owing to a combination of experimental detection noise and instabilities of the material under the electron beam as shown in Fig. 1(c). Although such histograms are often interpreted visually, determining the peak positions in this manner is highly subjective and strongly depends on the arbitrarily chosen number of bins to visualize the histogram. To avoid this, a statistics-based approach is suggested within which the originally estimated intensities are used rather than a histogram as such. The intensities are then regarded as independent statistical draws from a so-called Gaussian mixture model $^{9}$ :

$$
p_{\text {mix }}\left(V_{i} \mid \Psi_{G}\right)=\sum_{g=1}^{G} \pi_{g} \frac{1}{\sqrt{2 \pi} \sigma} \exp \left[\frac{-\left(V_{i}-\mu_{g}\right)^{2}}{2 \sigma^{2}}\right]
$$

defining the probability that a specific intensity value $V_{i}$ would be estimated for a particular atomic column $i$. The vector $\Psi_{G}=\left(\pi_{1}, \ldots, \pi_{G-1}, \mu_{1}, \ldots, \mu_{G}, \sigma\right)^{T}$ contains the unknown parameters of this probability distribution. The mixing proportions $\pi_{g}$ define the probability that a column contains $g$ atoms with $g$ ranging between 1 and a maximum number $G$, which in general is not known beforehand. Since every column should be assigned a number between 1 and $G$, the mixing proportions sum to unity so that one of the mixing proportions is redundant and only $G-1$ mixing proportions need to be determined. The parameters $\mu_{g}$ and $\sigma$ represent the position and standard deviation of the Gaussian components, 
respectively. The position $\mu_{g}$ corresponds to the mean intensity value of a column containing $g \mathrm{~Pb}$ atoms, whereas $\sigma$ describes the fluctuation of the estimated intensities about their mean value, thus accounting for inherent experimental uncertainties. It is important to note that within this model it has only been assumed that the intensity $\mu_{g}$ will increase with increasing number of atoms without imposing a particular intensity variation. As such, deviations from a linear intensity increase due to quantum mechanical channeling effects are implicitly taken into account. Next, the parameters $\Psi_{G}$ need to be estimated from the obtained intensities $V_{i}$. Because of its optimal statistical properties, the maximum likelihood estimator will be used. The parameter estimates are thus given by the values of $\Psi_{G}$ for which the likelihood function $L\left(\Psi_{G}\right)$ is maximum. For independent data $V_{i}$, the likelihood function is given by

$$
L\left(\Psi_{G}\right)=\prod_{i=1}^{I} p_{\text {mix }}\left(V_{i} \mid \Psi_{G}\right) .
$$

In general this maximization requires the use of an iterative numerical algorithm. For a given number of components $G$, the so-called expectation maximization algorithm is very effective. ${ }^{9}$ In practice, however, the value of $G$ is unknown and has to be inferred from the available data as well. For a hierarchic set of models $p_{\text {mix }}\left(V_{i} \mid \Psi_{G}\right)$, where an increase of the model order $G$ by 1 corresponds to an extra Gaussian component, the model fit will typically improve. Indeed, when more parameters are used, more details in the available realization $V_{i}(i=1, \ldots, I)$ are described. However, for highorder models, these details are random. In a new realization (when one would acquire a new image), other random details are found. Then, the estimated parameters do not provide useful information about the true stochastic process characteristics. As a result, the model quality, describing how well the estimated model describes the true process, will typically degrade with model order in this region. The true model order $G$ is found by balancing model fit against model quality. Therefore, the use of order selection criteria is needed. In a sense, such criteria evaluate the statistical significance of an extra component in the model. The so-called integrated classification likelihood $I C L$ criterion has been used since it outperforms other order selection criteria. ${ }^{9}$ It is defined as $-2 \log L\left(\Psi_{G}\right)+\left[2 E N\left(V_{i}, \Psi_{G}\right)+2 G \log I\right]$, where the function $E N\left(V_{i}, \Psi_{G}\right)$ estimates the entropy. The first term, measuring the model fit, will decrease with increasing order, whereas the term between squared brackets, measuring the complexity of the model, will increase with increasing order and will penalize high-order models. The estimated model order is given by the value of $G$ for which $I C L$ reaches a minimum. By means of simulations, we found out that this minimum often corresponds to a local optimum rather than to a global optimum. In Fig. 1(d) the $I C L$ criterion has been evaluated for $G$ varying between 1 and 40 showing a clear local minimum at 25. This suggests that a particular column contains 1 up to a maximum of $25 \mathrm{~Pb}$ atoms. The black solid curve in Fig. 1(c) shows the estimated mixture model; the individual Gaussian components are shown by means of red dashed curves. Based on the estimated probability distribution, the number of $\mathrm{Pb}$ atoms for a particular atomic column $i$ can be determined by computing the value of $g$ for which the probability that it generates the estimated intensity $V_{i}$ is largest. The results are summarized in Fig. 1(e) showing the number of $\mathrm{Pb}$ atoms for every column of the particle.

The technique has been found to be accurate and robust. A convenient way to assess the robustness and practical
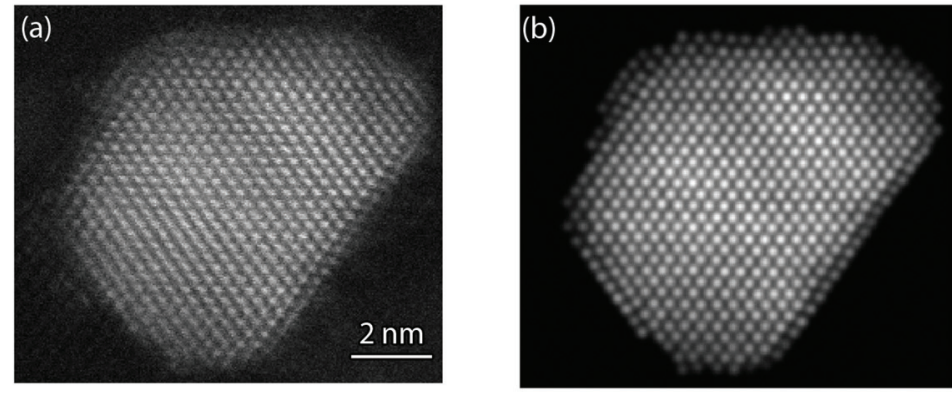

(c)

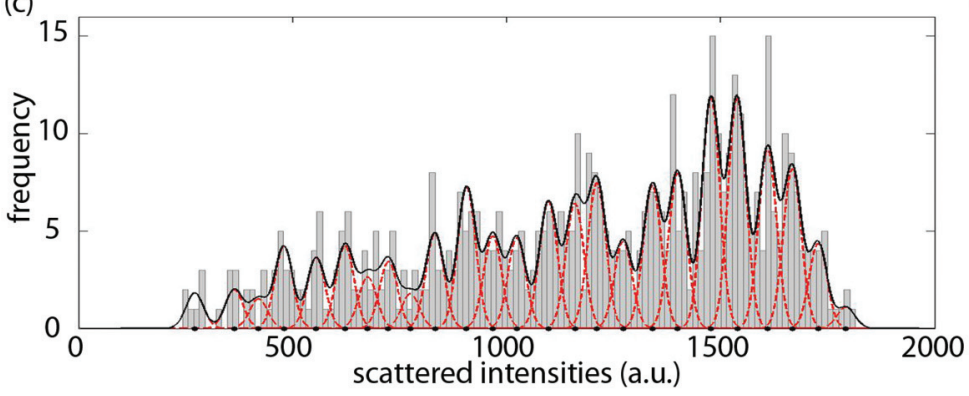

(e)

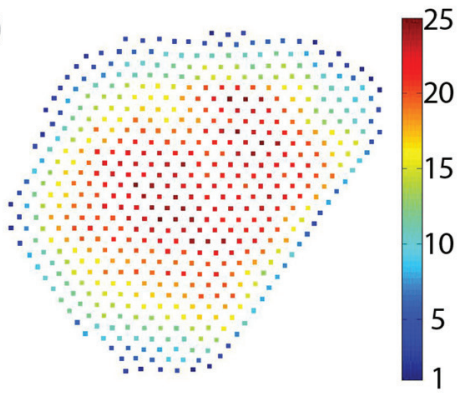

(d)

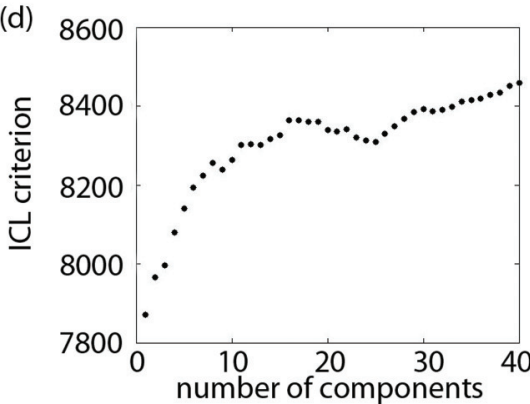

FIG. 1. (Color online) (a) Experimental HAADF STEM image of a Pb nanoparticle embedded in crystalline Si. (b) Refined model. (c) Histogram of scattered intensities of the Pb columns. The black solid curve shows the estimated mixture model; the individual components are shown as red dashed curves. (d) The integrated classification likelihood criterion evaluated as a function of the number of Gaussian components in a mixture model. (e) Number of $\mathrm{Pb}$ atoms per column. 
limitations of the method is by estimating from artificial, simulated intensities. Given a set of input parameters defining a Gaussian mixture model, a set of independent intensity draws $V_{i}(i=1, \ldots, I)$ is generated from this probability distribution. These data have then been used to find out if the $I C L$ criterion reveals a minimum at the correct number of components and to compare the estimated parameters with the known input parameters of the Gaussian mixture model. As a first test of the reliability of the method, we used the Gaussian mixture model derived from the experimental column intensities for the embedded $\mathrm{Pb}$ nanoparticle [shown in Figs. 1(c) and 2(a)]. This probability distribution consists of 25 components and has been used to simulate the intensity values shown in Fig. 2(a). The number of statistical draws has been chosen equal to 533 being in agreement with the number
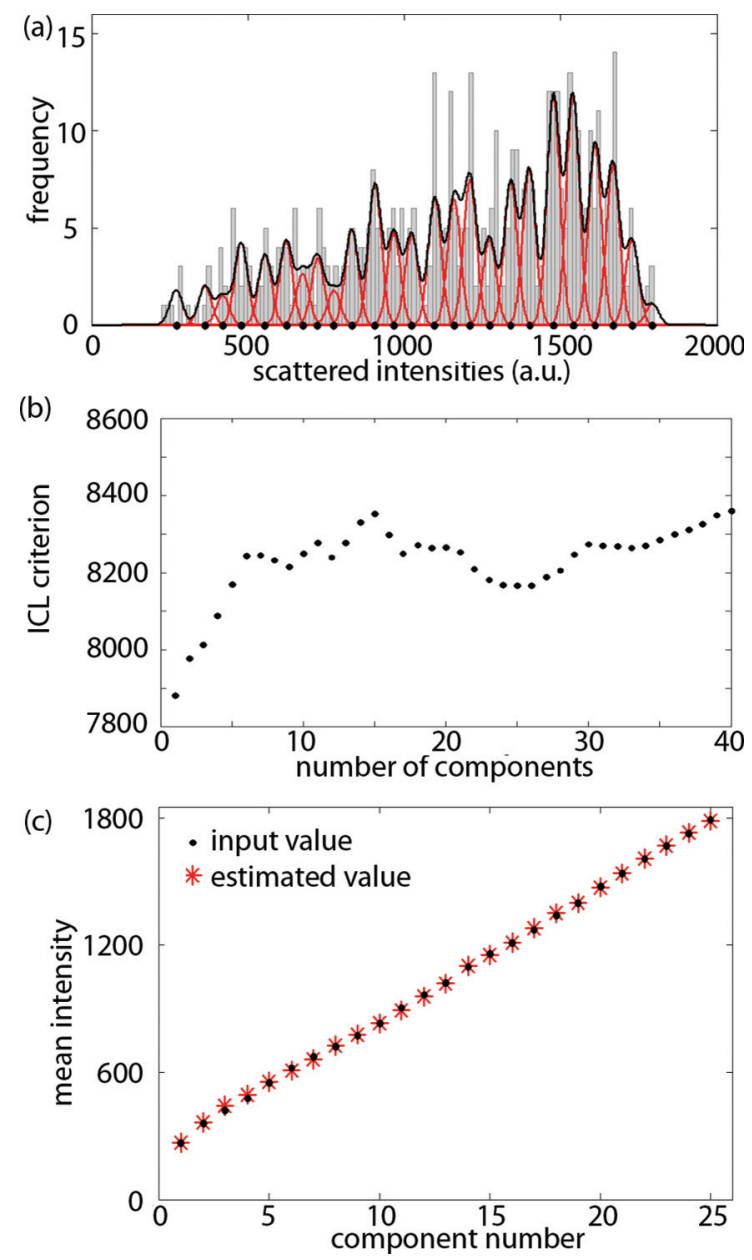

FIG. 2. (Color online) (a) Histogram of simulated intensities together with the underlying known Gaussian mixture model that has been used to generate these intensities. In this simulation, the input mixture model corresponds to the estimated mixture model shown in Fig. 1(c). The 25 true values for the mean intensities of each component are indicated by means of black dots. (b) The integrated classification likelihood criterion evaluated as a function of the number of Gaussian components in a mixture model. A clear minimum is revealed at 25 corresponding to the correct number of components. (c) Comparison of the input mean values for the simulation [corresponding to the black dots in (a)] together with the estimated parameters in red. of $\mathrm{Pb}$ columns in the experimental HAADF STEM image [see Fig. 1(a)]. Based on these simulated intensity values, the ICL criterion has been computed as shown in Fig. 2(b). A minimum is revealed at 25 corresponding to the correct number of components. In Fig. 2(c) we compare the input values for the mean intensities with the estimated intensities. A nearly perfect agreement is obtained proving the high accuracy and precision of the method in this example. Also in case of more components, the number of components as well as the mean component intensities are estimated with high reliability. This is shown in Figs. 3(a)-3(c) in which the input mixture model corresponds to the estimated mixture model following from experimental data of a Au nanorod that will be further discussed [see Fig. 7(c)]. The mean intensities of all 47 input mean intensities have been estimated with high accuracy and precision. In a similar way, it has been investigated if a nonlinear dependence of the intensities as a function of
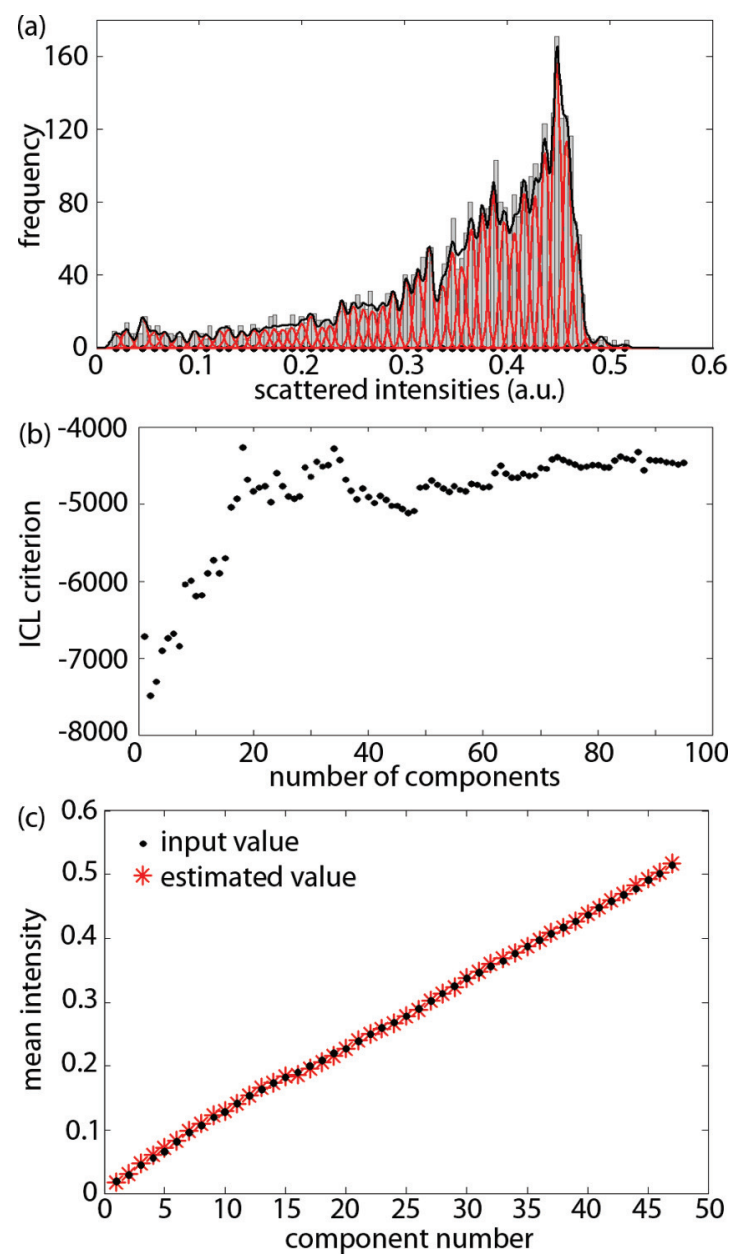

FIG. 3. (Color online) (a) Histogram of simulated intensities together with the underlying known Gaussian mixture model that has been used to generate these intensities. The 47 true values for the mean intensities of each component are indicated by means of black dots. (b) The integrated classification likelihood criterion evaluated as a function of the number of Gaussian components in a mixture model. A clear minimum is revealed at 47 corresponding to the correct number of components. (c) Comparison of the input mean values for the simulation [corresponding to the black dots in (a)] together with the estimated parameters in red. 

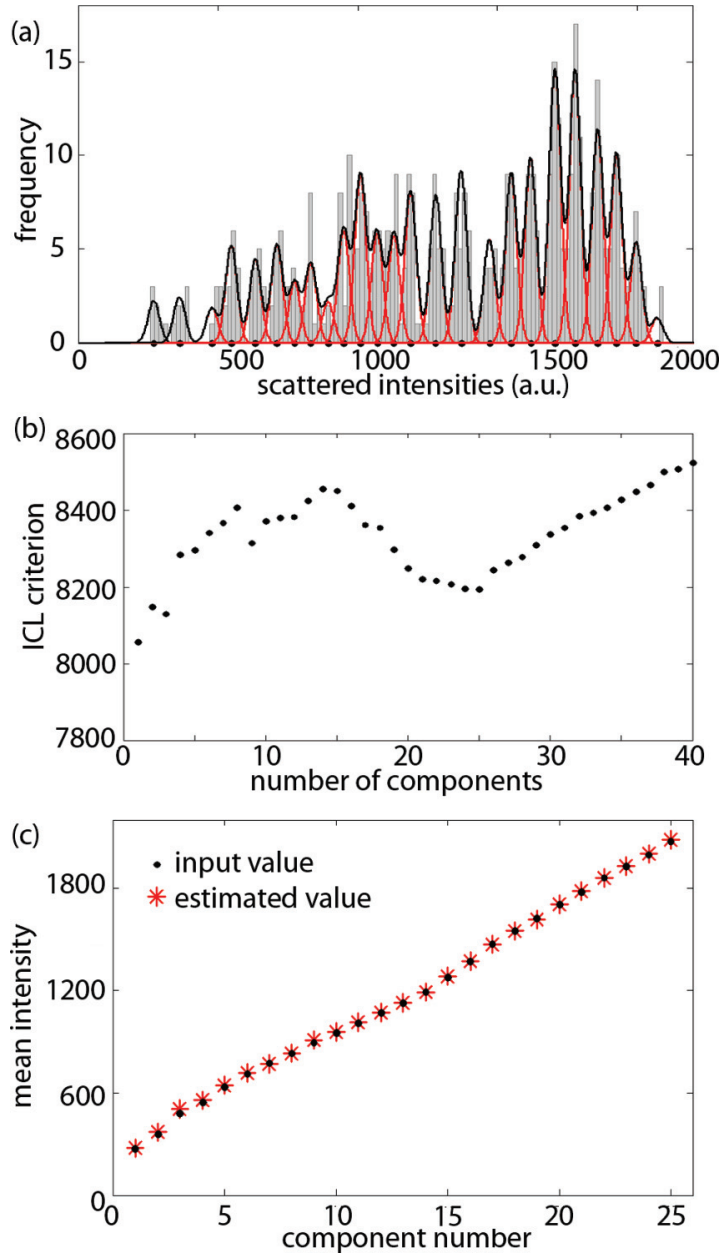

FIG. 4. (Color online) (a) Histogram of simulated intensities together with the underlying known Gaussian mixture model that has been used to generate these intensities. The 25 true values for the mean intensities of each component are indicated by means of black dots. Here a nonlinear increase of the intensities with increasing number of atoms has been assumed. (b) The integrated classification likelihood criterion evaluated as a function of the number of Gaussian components in a mixture model. A clear minimum is revealed at 25 corresponding to the correct number of components. (c) Comparison of the input mean values for the simulation [corresponding to the black dots in (a)] together with the estimated parameters in red.

the number of atoms is correctly determined. Although the experimental examples shown in this article seem to suggest a nearly linear increase, it is important to demonstrate that the method may account for nonlinearities if these are present in the original data. In Figs. 4(a)-4(c) a nonlinear increase of the intensities with increasing number of atoms has been assumed. Following the same procedure as before, the results show that nonlinearities are retrieved by the method and that the outcome is not forced to a linear dependence. The most important limitation is set in case the intensities would reach a plateau at higher number of atoms. In that case, there is strong overlap between neighboring components such that fewer Gaussians can be used to describe the experimental data. The simulation results summarized in Figs. 5(a)-5(c), in which it is assumed that the intensities reach a plateau at a thickness of 10 atoms, indeed reveal an underestimation of the
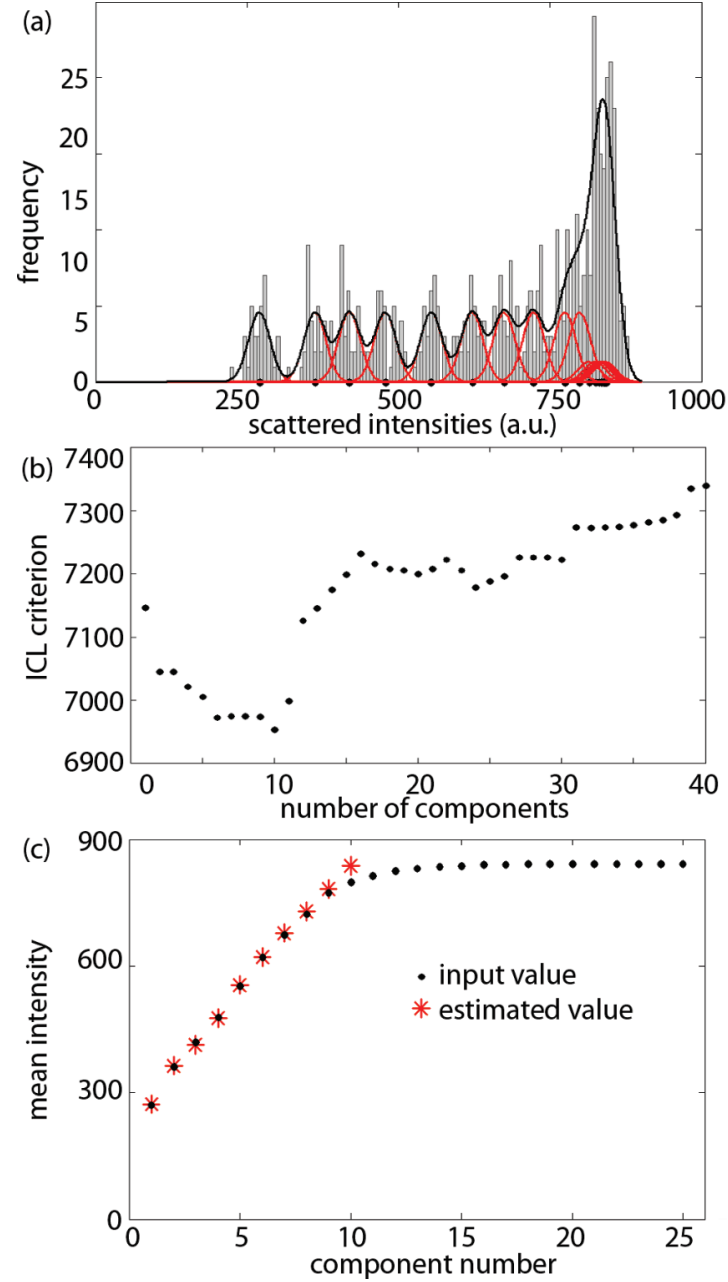

FIG. 5. (Color online) (a) Histogram of simulated intensities together with the underlying known Gaussian mixture model that has been used to generate these intensities. The 25 true values for the mean intensities of each component are indicated by means of black dots. In this simulation, it is assumed that the intensities reach a plateau at a thickness of 10 atoms. (b) The integrated classification likelihood criterion evaluated as a function of the number of Gaussian components in a mixture model. A clear minimum is revealed at 10 corresponding to an underestimation of the number of components. (c) Comparison of the input mean values for the simulation [corresponding to the black dots in (a)] together with the estimated parameters in red.

ICL criterion. Although the mean intensities of components 1 up to 9 are correctly determined, components 10 up to 25 are described by a single component. However, as will be demonstrated further, the proposed total intensity measure $V_{i}$ is robust and does not seem to level off as a function of thickness.

Whereas the previous experimental example shows the possibility to count heavy $\mathrm{Pb}$ atoms, the same elegant procedure can also be applied to lighter atoms. To illustrate this, three consecutive image frames of an ultrasmall Ge cluster acquired using a FEI TITAN operated at $120 \mathrm{kV}$ have been analyzed. From these images, shown in Figs. 6(a)-6(c), it is directly clear that such clusters undergo structural changes because of knock-on energy transfer from the electron beam. The dynamical behavior of such clusters can be studied from 

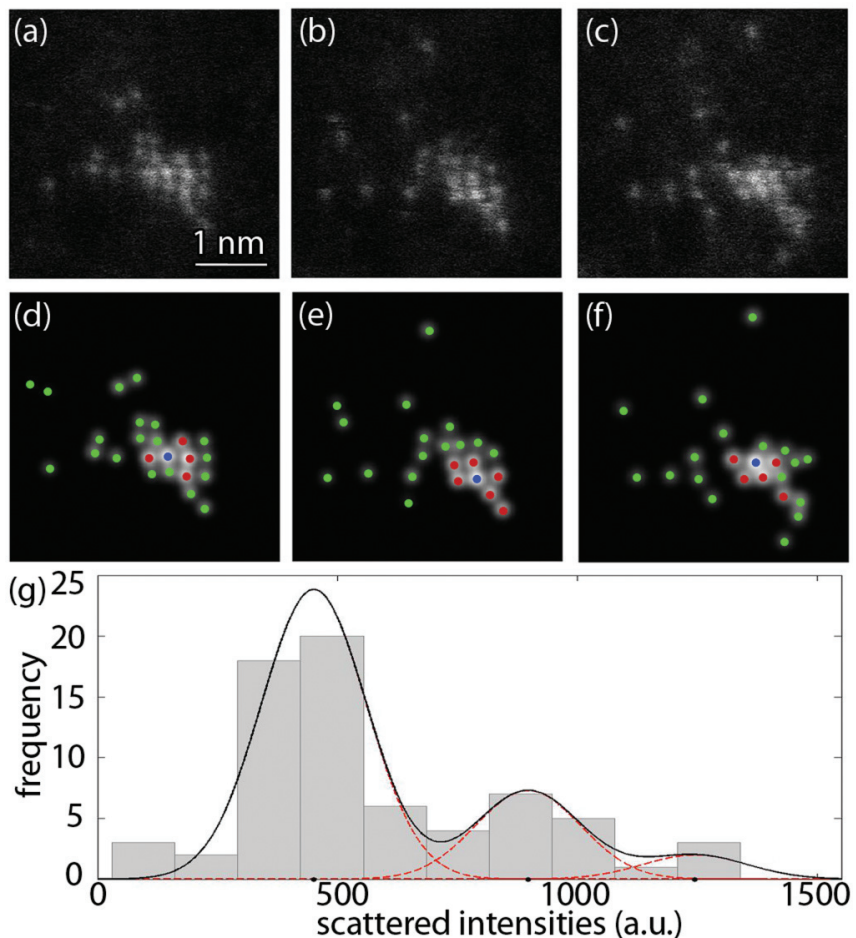

FIG. 6. (Color online) (a)-(c) Sequence of HAADF STEM images of a Ge cluster. (d)-(f) Refined models together with the counting results in which green, red, and blue dots correspond to one atom, two atoms, and three atoms, respectively. (g) Histogram of scattered intensities of the Ge atoms. The black solid curve shows the estimated mixture model; the individual components are shown as red dashed curves.

counting results obtained from each frame..$^{10}$ Refined models, using statistical parameter estimation theory, are shown in Figs. 6(d)-6(f). The scattered intensities are presented in Fig. 6(g). Evaluation of the $I C L$ criterion indicates the presence of three components from which we conclude that the estimated peaks correspond to one, two, or three Ge atoms projected on top of each other. The resulting counting results are indicated in Figs. 6(d)-6(f). It is important to note that the total number of atoms is conserved from frame to frame, proving the validity of the results obtained.

Also larger particles, such as the Au nanorod shown in Fig. 7(a), can be analyzed along the same lines. This nanorod has been imaged along the [100] zone axis using a double aberration-corrected FEI TITAN operated at $300 \mathrm{kV}$. The ICL criterion, shown in Fig. 7(b), reveals the presence of 47 different column types each consisting of a specific number of atoms. The histogram of scattered intensities is shown in Fig. 7(c) together with the estimated probability distribution. The number of Au atoms for every column of the nanorod is shown in Fig. 7(d). As expected for this nanorod, the thickness that can be derived from this atom counting analysis along the perpendicular direction matches the diameter obtained from Fig. 7(a). In this example, the intensities have been normalized with respect to the incident beam by taking the detector sensitivity into account. ${ }^{11,12}$ This places STEM experiments on the same absolute intensity scale as used in theoretical image simulations. As such, we can test the accuracy of the
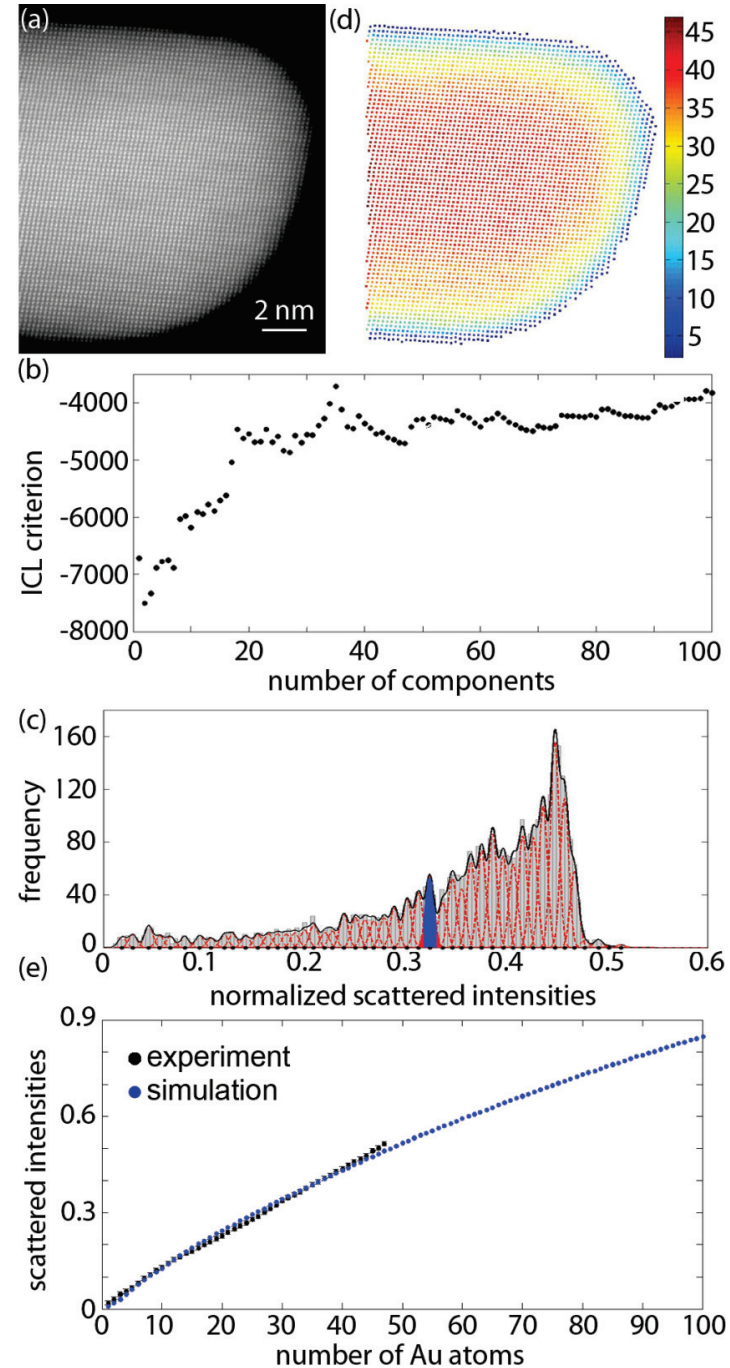

FIG. 7. (Color online) (a) HAADF STEM image of a Au nanorod. (b) The integrated classification likelihood criterion evaluated as a function of the number of Gaussian components in a mixture model. (c) Histogram of scattered intensities of the Au columns together with the estimated mixture model and its individual components. The component for which $g$ equals 29 is shown in color. Columns whose intensities belong to the central blue part will be assigned 29 atoms. Columns whose intensities belong to the outermost red part will be assigned 28 or 30 atoms. (d) Number of Au atoms per column. (e) Comparison of experimental and simulated scattered intensities together with $90 \%$ tolerance intervals.

counting procedure. Figure 7(e) shows the experimental mean intensities [corresponding to the peak positions in Fig. 7(c)] together with the scattered intensities $V_{i}$ estimated from frozen phonon calculations using the STEMsim program ${ }^{13}$ under the same experimental conditions. The excellent match of the experimental and simulated intensities within the expected $5 \%-10 \%$ error range ${ }^{5,6}$ validates the accuracy of the obtained atom counts. Minor deviations can indeed not be avoided because of remaining uncertainties in the microscope settings and practical limitations to fully take the complex dependence of the Debye-Waller factor on both the particle's size ${ }^{14}$ and its different behavior for surface atoms as compared to bulklike 
atoms ${ }^{15}$ into account. However, from an alternative point of view, the experimental mean intensities can be used as a reference to further improve the accuracy of imaging models taking all important physical effects into account. The steady increase of the simulated intensities even above 47 atoms proves the high sensitivity of the scattered intensities for the number of atoms. The precision, on the other hand, is limited by the unavoidable presence of noise in the experimental images resulting into fluctuations of the scattered intensities $V_{i}$ about the mean values $\mu_{g}$. The precision is then set by the overlap of the Gaussian components shown in Figs. 1(c), 6(g), and 7(c). When the overlap increases, the probability that a wrong number of atoms is assigned to a particular column indeed increases. From the examples discussed in this article, it is found that $86 \%, 93 \%$, and $80 \%$ of all atom columns are measured without error for the $\mathrm{Pb} / \mathrm{Si}, \mathrm{Ge}$, and $\mathrm{Au}$ case, respectively. The probability to miscount the number of atoms with \pm 1 equals $13 \%, 6 \%$, and $20 \%$, respectively, whereas the probability to have an error of \pm 2 atoms is almost zero in all examples discussed here. The procedure on how to compute these probabilities is illustrated in Fig. 7(c) in which the estimated Gaussian mixture model for the Au nanorod example is shown. Component $g=29$ is shown in color. This distribution will be generated by columns having 29 atoms in a column. In practice, all columns whose intensities belong to the central blue part of this component will be assigned the correct number of atoms (29 in this example). This fraction therefore corresponds to the probability to assign 29 atoms to columns having effectively 29 atoms in a column. On average, that is, by averaging fractions for the components 1 up to 47 , this probability corresponds to $80 \%$. Columns whose intensities belong to the outermost red part will be assigned either 28 or 30 atoms since it is more likely that these intensities would be generated by columns containing 28 or 30 atoms. On average, the probability to miscount the number of atoms with \pm 1 equals $20 \%$ in this example. Furthermore, since there is no overlap with second neighboring columns, the probability to miscount the number of atoms with \pm 2 equals $0 \%$. As compared to the results obtained when using simulations alone ${ }^{4}$ the amount of overlap with neighboring components is greatly reduced when using this model-based procedure, thus significantly improving the precision that can be achieved.

In conclusion, we present a procedure to count the number of atoms in a nanocrystal with trustworthy single-atom sensitivity. This method requires the application of advanced statistical methods to HAADF STEM images without the need for a priori knowledge about the atomic structure. The independence of the method from image simulations allowed us to test the reliability of the method. Furthermore, the performance and robustness have been tested in terms of accuracy and precision demonstrating counting results with an error of 1 atom at maximum. Several examples show its application to samples of arbitrary shape, size, and atom type.

The authors acknowledge financial support from the Research Foundation Flanders (FWO, Belgium) and a Ph.D. research grant to A.D.B and B.G. We acknowledge financial support from the European Union Seventh Framework Program under Grant Agreements No. 262348 ESMI, No. 312483 ESTEEM2, and ERC Grant No. 246791 COUNTATOMS. The authors acknowledge financial support from the Flemish Hercules 3 programme for large infrastructure. A.R. thanks the DFG under Contract No. RO2057/8-1. The authors are grateful to M. Van Bael and P. Lievens (KU Leuven) and to L. M. Liz-Marzán (University of Vigo) for providing the samples. *sandra.vanaert@ua.ac.be

${ }^{1}$ P. Hartel, H. Rose, and C. Dinges, Ultramicroscopy 63, 93 (1996).

${ }^{2}$ P. M. Voyles, D. A. Muller, J. L. Grazul, P. H. Citrin, and H.-J. L. Gossmann, Nature (London) 416, 826 (2002).

${ }^{3}$ Z. Y. Li et al., Nature (London) 451, 46 (2008).

${ }^{4}$ J. M. LeBeau, S. D. Findlay, L. J. Allen, and S. Stemmer, Nano Lett. 10, 4405 (2010).

${ }^{5}$ J. M. LeBeau, S. D. Findlay, L. J. Allen, and S. Stemmer, Phys. Rev. Lett. 100, 206101 (2008).

${ }^{6}$ A. Rosenauer et al., Ultramicroscopy 111, 1316 (2011).

${ }^{7}$ S. Van Aert, K. J. Batenburg, M. D. Rossell, R. Erni, and G. Van Tendeloo, Nature (London) 470, 374 (2011)

${ }^{8}$ S. Van Aert, J. Verbeeck, R. Erni, S. Bals, M. Luysberg, D. Van Dyck, and G. Van Tendeloo, Ultramicroscopy 109, 1236 (2009).
${ }^{9}$ G. McLachlan and D. Peel, Finite Mixture Models (John Wiley, Canada, 2000).

${ }^{10}$ S. Bals et al., Nat. Commun. 3, 897 (2012).

${ }^{11}$ A. Rosenauer, K. Gries, K. Müller, A. Pretorius, M. Schowalter, A. Avramescu, K. Engl, and S. Lutgen, Ultramicroscopy 109, 1171 (2009).

${ }^{12}$ T. Grieb, K. Müller, R. Fritz, M. Schowalter, N. Neugebohrn, N. Knaub, K. Volz, and A. Rosenauer, Ultramicroscopy 117, 15 (2012).

${ }^{13}$ A. Rosenauer and M. Schowalter, in Springer Proceedings in Physics: Microscopy of Semiconducting Materials (MSM) Conference, Cambridge, 2007, edited by A. G. Cullis and P. A. Midgley (Springer, Berlin, 2007), Vol. 120, pp. 169-172.

${ }^{14}$ P.-A. Buffat, Solid State Commun. 23, 547 (1977).

${ }^{15}$ B. C. Clark, R. Herman, and R. F. Wallis, Phys. Rev. 139, A860 (1965). 